\title{
Tokelau Language
}

National Cancer Institute

\section{Source}

National Cancer Institute. Tokelau Language. NCI Thesaurus. Code C154169.

A Polynesian language spoken in Tokelau and on Swains Island in American Samoa. 